\title{
Milligram Equivalent
}

National Cancer Institute

\section{Source}

National Cancer Institute. Milligram Equivalent. NCI Thesaurus. Code C156468.

A unit of relative amount of substance concentration equal to one thousandth of a gram

of an equivalent weight. 